\title{
Novel Food-Safe Spin-Lattice Relaxation Time Calibration Samples for Use in Magnetic Resonance Sensor Development ${ }^{+}$
}

\author{
Najlaa K. Almazrouei *, Michael I. Newton, Elizabeth R. Dye and Robert H. Morris \\ School of Science and Technology, Nottingham Trent University, Nottingham NG11 8NS, UK; \\ michael.newton@ntu.ac.uk (M.I.N.); elizabeth.dye2016@my.ntu.ac.uk (E.R.D.); rob.morris@ntu.ac.uk (R.H.M.) \\ * Correspondence: najlaa.almazrouei022016@my.ntu.ac.uk; Tel.: +44-11-5848-3315 \\ + Presented at the 4th International Electronic Conference on Sensors and Applications, 15-30 November \\ 2017; Available online: http://sciforum.net/conference/ecsa-4.
}

Published: 14 November 2017

\begin{abstract}
Magnetic Resonance (MR) sensors are an area of increasing interest for the measurement and monitoring of material properties. There are two relaxation times associated with samples that can be measured with MR sensors: The spin-lattice and spin-spin relaxations. When developing new sensors, it is desirable to have a series of standards by which instruments can be assessed. The standard calibration materials available typically comprise different concentrations of Nickel Sulphate which is carcinogenic and toxic. In this work we report the use of solutions containing full fat milk powder as a safe and inexpensive material that shortens the longitudinal relaxation time of water over a wide range of values. We demonstrate that concentrations in distilled water from $5 \%$ $w / v$ to $64 \% w / v$ give $\mathrm{T}_{1}$ values from $1.7 \mathrm{~s}$ down to $469 \mathrm{~ms}$ respectively in a $1.5 \mathrm{~T}$ clinical MRI, while within an MR sensor these times were from $1.6 \mathrm{~s}$ down to $431 \mathrm{~ms}$. In addition, both systems have the same exponential coefficient $(-0.022 \times$ concentration) that demonstrates the effectiveness of the NMR sensor in comparison to the clinical MRI.
\end{abstract}

Keywords: NMR; spin-lattice relaxation time; MRI

\section{Introduction}

Sensors based on the measurement of Magnetic Resonance (MR) relaxation times have been increasing in popularity, due in part to developments in permanent magnet technology. Such sensors typically measure the spin-lattice (longitudinal) relaxation time $\mathrm{T}_{1}$, or the effective spin-spin (transverse) relaxation time $\mathrm{T}_{2}{ }^{\text {eff }}[1-3]$. The molecular size, the physical and chemical environment of the nucleus, such as the viscosity of the solution affect the MR relaxation times, thus allowing determination of material properties. It is therefore important when developing such sensors, that there are a range of safe and repeatable reference samples to aid in calibration and testing. For the spin-spin relaxation times different viscosities of polydimethylsiloxane (PDMS) oil provide a suitable range of safe test materials [4,5]. However, for the spin-lattice relaxation times, available options are not as safe to use and typically consist of different concentrations of Nickel Sulphate, which is carcinogenic and toxic or Copper Sulfate solutions [6,7].

It has been previously shown that the water self-diffusion in dairy products depends on the amount of the protein, lactose and fat in the sample which also affect the relaxation times [8,9]. In this work we report the use of solutions comprising of full fat milk powder as a safe and inexpensive material that can affect the longitudinal relaxation time over a wide range of values and give a set of samples which are easily replicated in any setting. We demonstrate that concentrations in distilled 
water from $5 \% w / v$ to $64 \% w / v$ produce a wide range of $\mathrm{T}_{1}$ values. We validate the range of $\mathrm{T}_{1}$ values on a $1.5 \mathrm{~T}$ clinical MRI system and MR Sensor.

\section{Material and Methods}

\subsection{Sample Preparation}

Full fat milk powder (NIDO, Nestle, Vevey, Switzerland) and distilled water are all that is required to prepare the test samples. The milk powder was dissolved in distilled water at seven different concentrations $(5 \%, 11 \%, 18 \%, 23 \%, 38 \%, 50 \%$ and $64 \%$ all $w / v)$ within sample tubes and mixed thoroughly before the clinical MRI images were collected. The measurements made with the magnetic resonance sensor were collected using freshly prepared samples. Three of each sample were extracted and used to fill separate $5 \mathrm{~mm}$ glass, standard NMR tubes to collect average measurements for each concentration.

\subsection{MRI Measurement}

The first experiment demonstrates the suitability of the samples as a general $\mathrm{T}_{1}$ calibration sample, using a high field 1.5T clinical MRI system (Magnetom Avanto, Siemens, Munich, Germany) to verify that full fat milk powder does indeed affect the spin-lattice relaxation time $\left(\mathrm{T}_{1}\right)$. This took the form of the Acronym of Nottingham Trent University using selected wells of three 24 well plates. These were imaged using an inversion recovery pulse sequence with varying inversion times (100, 500, 750, 1000, 1250, 1500, 1750, 2000, 2250, 2500, 3000, 3500, 4000 and 4500) ms with a $5000 \mathrm{~ms}$ repetition time and coronal orientation. The spine coil was used in order to transmit and collect radiofrequency signals for these samples as they are inherently flat. The $T_{1}$ values was extracted using in house Matlab software (Math Works ${ }^{\circledast}$, Natick, MA, USA) to produce a T1 map, encoding the value of $\mathrm{T}_{1}$ in the colour scale.

\subsection{Magnetic Resonance Sensor Measurement}

The Magnetic Resonance sensor used was a customized closed loop, iron yoke magnet shown in Figure 1, generating a magnetic field of $308 \mathrm{mT}$, equivalent to a proton resonant frequency of 19.38 $\mathrm{MHz}$. For the transmitting and receiving radio frequency signal, an RF coil, as part of a commercial NMR Probe (Part 514 606, Leybold Didactic GmbH, Huerth, Germany) is positioned over the two permanent magnets. This ensures a homogenous field in the sample region. The sample was placed inside the coil for signal detection. This coil was connected to $\mathrm{Kea}^{2}$ spectrometer (Magritek, Wellington, New Zealand) through a standard series parallel tuning and matching circuit. The data was collected using Prospa (Version 3.22, Magritek, Wellington, New Zealand). The experimental measurements were taken using a $\mathrm{T}_{1}$ inversion recovery sequence, using seventeen interexperimental repetition times ranging from $25 \mathrm{~ms}$ to $6000 \mathrm{~ms}$ in non-uniform increments. The maximum delay time was set to $10,000 \mathrm{~ms}$ which it is seven times the $\mathrm{T}_{1}$ value estimated by the clinical MRI. Eight Echoes with the shortest achievable echo time were summed to improve the signal to noise ratio.

The data collected using the $\mathrm{Kea}^{2}$ were processed using an online program (MRI toolbox website, (c) 2011-13 Alan McMillan) [10]. The $T_{1}$ values were extracted from the mono-exponential fit of the signal intensity against repetition times.

\section{Results and Discussion}

The viscosity of a sample is well known to affect the $\mathrm{T}_{1}$ relaxation parameter. In this work we observe that as the viscosity increased, the spin lattice relaxation time values declined which is as expected. There is a relationship between the viscosity and the molecular size of the sample [10]. The sample is a full fat milk powder which involve protein, lactose and fat, where fat has a comparable molecular size as the protein. As these component increased in volume (by virtue of the concentration increase), the nucleus rotation will be slower and this causes a faster decay of the relaxation time $[9,11]$. 


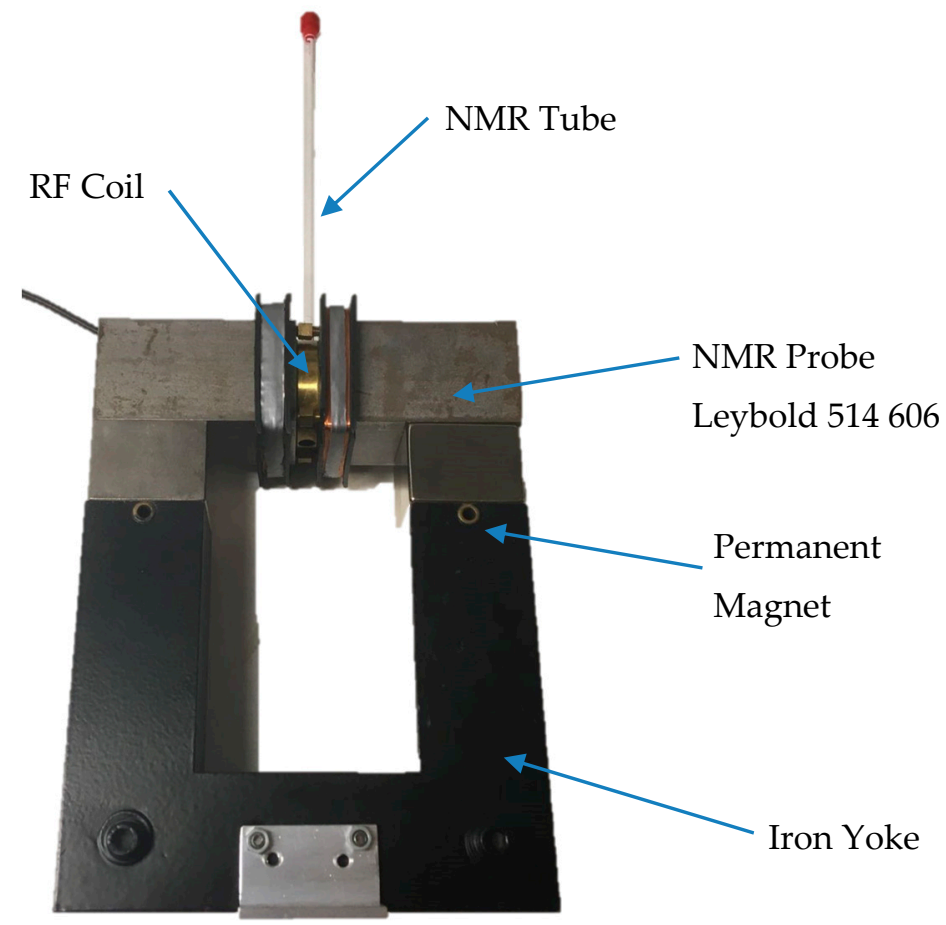

Figure 1. Photograph of closed Loop Magnet setup used for sensor measurements showing the permanent magnets, iron yoke and Leybold NMR Probe.

Figure 2 demonstrates the changes in the image contrast due to the changes in the inversion time $\left(\mathrm{T}_{\mathrm{i}}\right)$ applied and the different milk concentration on each part of the letters (NTU) using the spine coil. Samples measured using clinical MRI shows decreasing $\mathrm{T}_{1}$ values as the concentration is increased as shown in Figure 3 with the following average $\mathrm{T}_{1}$ values $(1.7 \pm 6.7,1.46 \pm 19.6,1.25 \pm 37.89,1.18 \pm$ $27.8,0.838 \pm 33.59,0.886 \pm 8.29$ and $0.469 \pm 13)$ seconds for the weight/volume concentrations of $(5,11$, $18,23,38,50,64)$ percent respectively.

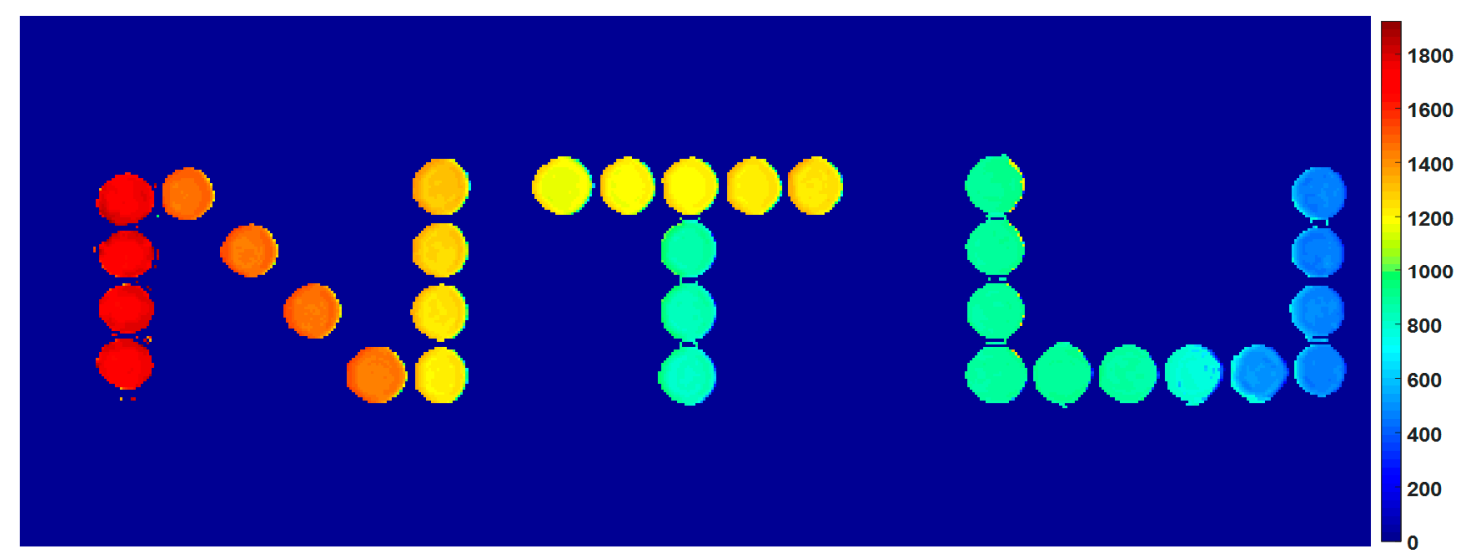

Figure 2. Inversion recovery $\mathrm{T}_{1}$ map of different milk concentrations increasing from left to right, four of each sample. The color bar scale is $\mathrm{T}_{1}$ in $\mathrm{ms}$. 


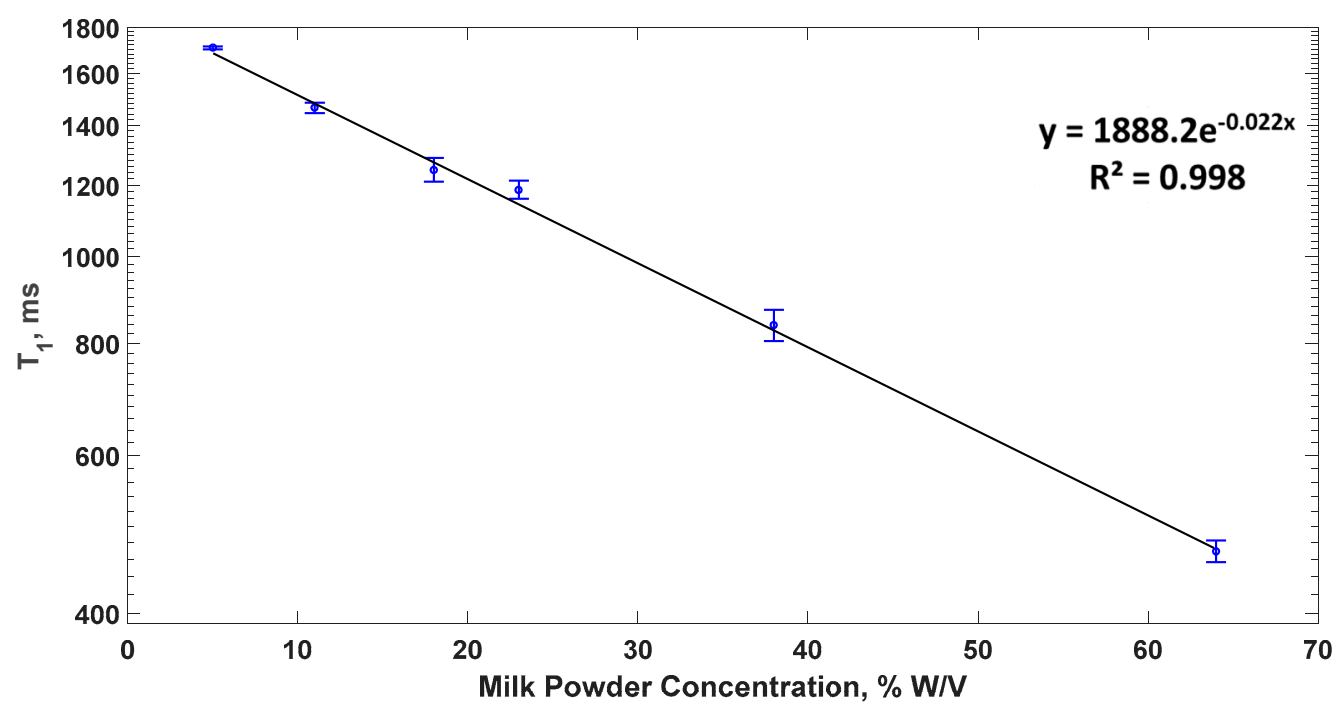

Figure 3. Spin lattice relaxation time $\mathrm{T}_{1}$ values against full fat milk powder concentration measured using clinical MRI using the spine matrix coil.

These concentrations were freshly prepared and measured using the customized NMR sensor. All the measurements were performed inside a bench top Faraday cage to reduce the external noise. The values of $\mathrm{T}_{1}$ were averaged from the individual measurements of three samples for each concentration. Figure 4 illustrates the relationship between the $\mathrm{T}_{1}$ values against the milk concentration. The $\mathrm{T}_{1}$ values that were generated were $(1.65 \pm 70.7,1.32 \pm 43.62,1.11 \pm 39,0.939 \pm 26.26$, $0.724 \pm 22.6,0.539 \pm 21.5$ and $0.431 \pm 5.34)$ second for the Weight/Volume concentrations of $(5,11,18$, $23,38,50,64)$ percent respectively. The errors bars were taken from the standard deviation of these values, to show the repeatability from sample to sample.

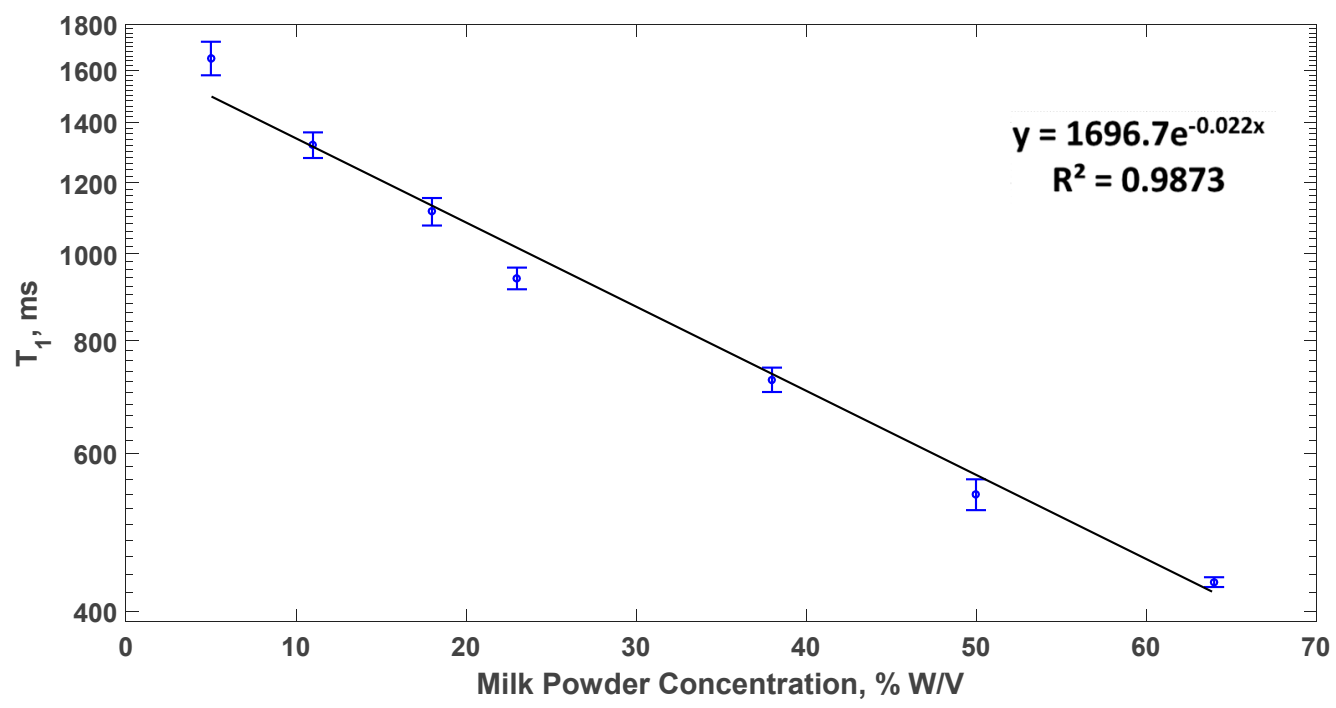

Figure 4. Spin lattice relaxation time $\mathrm{T}_{1}$ values against full fat milk powder concentration measured using a customized MR sensor. The error bars show the standard deviation of the measurements.

It is interesting to note that despite the high difference in the intensity of magnetic field between the clinical MRI (1.5 Tesla) and the MR sensor $(308 \mathrm{mT})$, the $\mathrm{T}_{1}$ relaxation of the full fat milk powder samples was similar. This is well demonstrated by observing the exponential fitting coefficient which in both systems is $-0.022 \times$ concentration. This demonstrates the effectiveness of full fat milk powder samples as calibration standards for the sensor development. 


\section{Conclusions}

The different concentrations of full fat milk powder show an excellent relationship with the spinlattice relaxation time. Both the clinical MRI scanner and the MR sensor have the same exponential fitting coefficient that demonstrates well that full fat milk powder in distilled water is a safe, inexpensive material that can be used as a tool for MR sensor development. We recommend that freshly prepared sample should be used between $0-4 \mathrm{~h}$ to avoid the fluctuation in the $\mathrm{T}_{1}$ values due to the separation that can occur to avoid needing to add a stabilizer that could affect the relaxation times. The ease of preparation, low cost and availability of the material are ideal for such a system.

Acknowledgments: NKA acknowledges the Ministry of Higher Education and Scientific Research (MOHESR) in the United Arab Emirates for funding, and the Dubai Health Authority (DHA) for study leave.

Author Contributions: Robert H. Morris and Michael I. Newton conceived, designed the experiments, reviewed and edited the manuscript; Elizabeth R. Dye produced the software used for $\mathrm{T}_{1}$ experiments with the Kea ${ }^{2}$, Najlaa K. Almazrouei performed the experiments, analyzed the data and wrote the manuscript.

Conflicts of Interest: The authors declare no conflict of interest.

\section{References}

1. Blümich, B.; Perlo, J.; Casanova, F. Mobile single-sided NMR. Prog. Nucl. Magn. Reson. Spectrosc. 2008, 52, 197-269.

2. Manz, B.; Coy, A.; Dykstra, R.; Eccles, C.D.; Hunter, M.W.; Parkinson, B.J.; Callaghan, P.T. A mobile onesided NMR sensor with a homogeneous magnetic field: The NMR-MOLE. J. Magn. Reson. 2006, 183, 25-31.

3. Blümich, B.; Casanova, F.; Appelt, S. NMR at low magnetic fields. Chem. Phys. Lett. 2009, 477, 231-240.

4. Cosgrove, T.; Roberts, C.; Garasanin, T.; Schmidt, R.G.; Gordon, G.V. NMR Spin-Spin relaxation studies of silicate-filled low molecular weight poly (dimethylsiloxane)s. Langmuir 2002, 18, 10080-10085.

5. Simon, G.; Birnstiel, A.; Schimmel, K. Network characterisation of end-linked poly (dimethylsiloxane) by 1 H-NMR-spin-spin relaxation. Polym. Bull. 1989, 21, 235-241.

6. Christoffersson, J.O.; Olsson, L.; Sjöberg, S. Nickel-doped agarose gel phantoms in MR imaging. Acta Radiol. 1991, 32, 426-431.

7. Mitchell, M.D.; Kundel, H.L.; Axel, L.; Joseph, P.M. Agarose as a tissue equivalent phantom material for NMR imaging. Magn. Reson. Imaging 1986, 4, 263-266.

8. Métais, A.; Cambert, M.; Riaublanc, A.; Mariette, F. Effects of casein and fat content on water self-diffusion coefficients in casein systems: A pulsed field gradient nuclear magnetic resonance study. J. Agric. Food Chem. 2004, 52, 3988-3995.

9. Todt, H.; Guthausen, G.; Burk, W.; Schmalbein, D.; Kamlowski, A. Water/moisture and fat analysis by timedomain NMR. Food Chem. 2006, 96, 436-440.

10. MRI Toolbox Website. Available online: http://www.mritoolbox.com/ParameterFitting.htm1 (accessed on 19 October 2017).

11. Colsenet, R.; Mariette, F.; Cambert, M. NMR relaxation and water self-diffusion studies in whey protein solutions and gels. J. Agric. Food Chem. 2005, 53, 6784-6790. 